worse outcome than those with PH-COPD ( $p=0.015)$, and borderline worse outcome than patients with PH-ILD $(\mathrm{p}=0.050)$, Figure. 48 of 94 patients were diagnosed with severe PH-RESP, defined at $\mathrm{mPAP} \geq 40 \mathrm{mmHg}$. WHO functional class $(\mathrm{p}=0.036)$, TLCO $(p=0.019)$, RVEF ( $p=0.033$ ) were significant independent predictors of outcome in patients with severe PH-RESP.

Conclusion Patients with severe PH-RESP have a dire clinical outcome. RVEF is an independent predictor of adverse outcome in these patients and may be a powerful biomarker for use in clinical trials of targeted therapy in patients with pulmonary hypertension associated with lung disease, particularly given the unreliable performance of echocardiography in patients with advanced lung disease.

\section{S121 THE UTILITY OF THE INCREMENTAL SHUTTLE WALKING TEST IN PULMONARY HYPERTENSION: RESULTS FROM THE ASPIRE REGISTRY}

CG Billings, J Hurdman, R Condliffe, I Armstrong, I Smith, CA Elliot, DG Kiely. Sheffield Pulmonary Vascular Disease Unit, Sheffield, UK

10.1136/thoraxjnl-2014-206260.127

Introduction The Incremental Shuttle Walk Test (ISWT) has been evaluated in a number of diseases and found to be a safe, reproducible test which correlates better with $\mathrm{VO}_{2}$ max than the Six-Minute Walk Test (6MWT). We aimed to evaluate the utility of the ISWT as a prognostic indicator in pulmonary hypertension (PH).

Methods Data was retrieved for consecutive cases of $\mathrm{PH}$ diagnosed in our unit from 2001-2010, a cohort previously described. ${ }^{1}$ ISWT was performed routinely as part of baseline assessment according to a modified protocol of Singh et al. ${ }^{2}$ Data was analysed in 5 Groups

\section{Figure I. Cumulative survival by Incremental Walk Test distance}

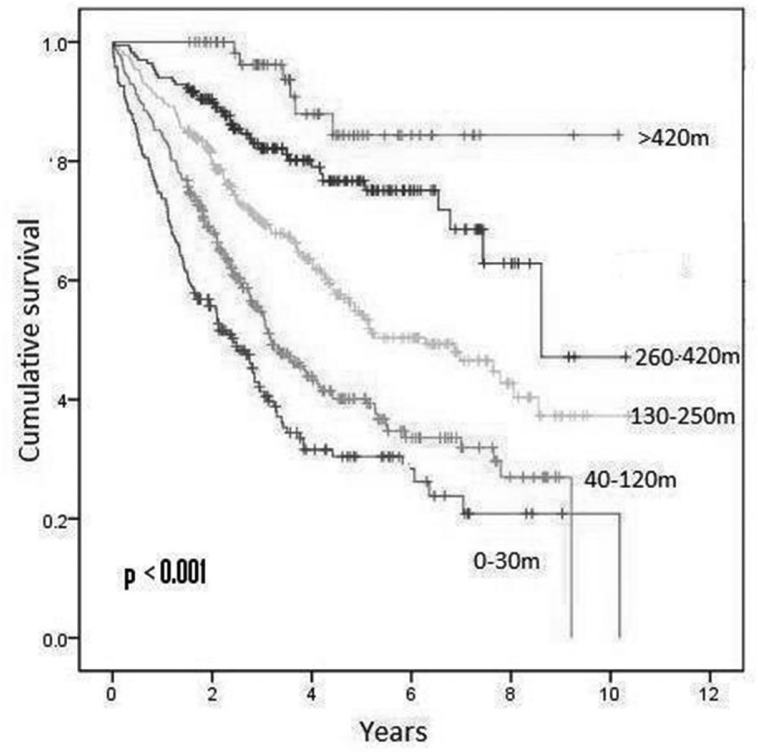

$\begin{array}{lllllllllll}0-30 \mathrm{~m} & 191 & 139 & 94 & 52 & 31 & 21 & 13 & 8 & 4 & 2 \\ 40-120 \mathrm{~m} & 297 & 251 & 181 & 119 & 74 & 51 & 29 & 18 & 9 & 1 \\ 130-250 \mathrm{~m} & 278 & 251 & 199 & 144 & 111 & 74 & 5 ? & 32 & 19 & 7 \\ 260-420 \mathrm{~m} & 169 & 159 & 134 & 98 & 69 & 49 & 29 & 20 & 9 & 3 \\ \text { s } 420 \mathrm{~m} & 63 & 63 & 63 & 42 & 29 & 16 & 10 & 6 & 2 & 2 \\ \text { Totals } & 998 & 863 & 671 & 455 & 314 & 211 & 138 & 84 & 43 & 15\end{array}$

Abstract S121 Figure 1 Cumulative survival by Incremental Walk Test distance according to the distance achieved based on ISWT level. A p-value of was deemed statistically significant.

Results 1002 of 1,344 patients diagnosed with $\mathrm{PH}$ underwent baseline ISWT within 3 months of cardiac catheterization and prior to pulmonary vascular therapy. Complete baseline data was available for 998 patients.

Kaplan-Meier analysis showed that increasing level of ISWT was associated with increased survival (Figure 1), including the PAH sub-group, with no ceiling effect.

ISWT distance correlated with WHO Functional Class, right atrial pressure, pulmonary vascular resistance, cardiac index, mixed venous oxygen saturation and percent predicted carbon monoxide diffusion (DLco) ( $\mathrm{p}$ all $\leq 0.01$ ). Multivariate Cox regression survival analysis including sex, body mass index, age, haemodynamic parameters and percent predicted DLco, demonstrated that ISWT distance was an independent predictor of survival.

One year follow-up data was available for 397 patients. KaplanMeier analysis showed that ISWT level on treatment at 1 year was predictive of survival $(\mathrm{p}<0.001)$. Survival was also superior in patients whose ISWT distance improved from baseline $\geq 30 \mathrm{~m}$ compared to those whose distance remained stable $(-20$ to $+20 \mathrm{~m})$ or declined by $\geq 30 \mathrm{~m}(\mathrm{p}=0.20)$.

Conclusion Baseline ISWT distance correlates with WHO functional class and pulmonary haemodynamics with no ceiling effect. It is an independent predictor of survival and change in ISWT predicts outcome. These features make it a viable alternative to the 6MWT in the assessment of patients with pulmonary hypertension, with a number of potential advantages.

\section{REFERENCES}

1 Hurdman et al. Eur Respir J 2012:39:945-955

2 Singh et al Thorax 1992:47:1019-1024

\section{S122 OUTCOME AFTER PULMONARY ENDARTERECTOMY (PEA): LONG TERM FOLLOW-UP OF THE UK NATIONAL COHORT}

${ }^{1} \mathrm{~J}$ Cannon, ${ }^{2} \mathrm{~L}$ Su, ${ }^{1} \mathrm{~K}$ Page, ${ }^{1} \mathrm{~A}$ Ponnaberanam, ${ }^{1} \mathrm{M}$ Toshner, ${ }^{1} \mathrm{D}$ Taboada, ${ }^{1} \mathrm{~K}$ Sheares, ${ }^{1} \mathrm{C} \mathrm{Ng},{ }^{1}$ J Dunning, ${ }^{1} \mathrm{~S}$ Tsui, 'D Jenkins, 'J Pepke-Zaba. 'Papworth Hospital, Cambridge, UK; ${ }^{2}$ MRC Institute of Public Health, Cambridge, UK

\subsection{6/thoraxjnl-2014-206260.128}

Introduction Chronic thromboembolic pulmonary hypertension (CTEPH) is a life threatening condition that historically has a poor outcome with supportive medical treatment. Pulmonary endarterectomy (PEA) is the treatment of choice and offers the only chance of cure. Data on the predictors of long term survival after PEA are limited. We analysed the long-term data from the UK PEA cohort.

Method All patients who underwent a PEA for CTEPH at Papworth hospital between January 1997 and December 2012 were included. Pre- and post-operative data on haemodynamics, exercise capacity, functional class and targeted $\mathrm{PAH}$ therapies taken were obtained from databases of the UK PH centres. The NHS spine summary care record tracking system was used for survival data and causes of death from the England and Scotland General Register Offices. The causes of death were further classified into 4 groups: 1. Post operative, 2. Right ventricular failure away from operative period, 3. Related to anticoagulation, 4. Unrelated to CTEPH e.g. malignancy.

Results 880 patients underwent PEA over the 15 year period. The mean age was 57 (range 15-84) and 53\% were male. 89\% were in WHO functional class 3 or 4 before surgery with an mean mPAP of $47 \mathrm{mmHg}$ and PVR of 830 dynes. Post surgery $84 \%$ of patients 